\title{
Enhanced light trapping in realistic thin film solar cells using one- dimensional gratings
}

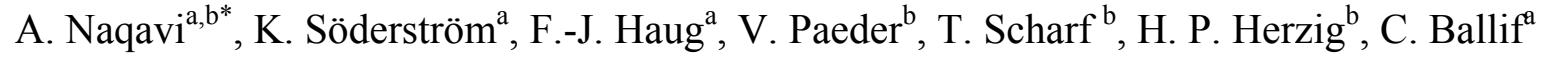 \\ ${ }^{a}$ Photovoltaics and Thin Film Electronics Laboratory, Institute of Microengineering (IMT), Ecole \\ Polytechnique Fédérale de Lausanne (EPFL), Rue A.-L. Breguet 2, 2000 Neuchâtel, Switzerland \\ ${ }^{b}$ Optics and Photonics Technology Laboratory, Ecole Polytechnique Fédérale de Lausanne (EPFL), \\ Rue A.-L. Breguet 2, 2000 Neuchâtel, Switzerland
}

\begin{abstract}
Finding the optimal structure to enhance light trapping in thin film silicon solar cells has attracted much attention in the previous decades. However, because of problems in integrating theory and experiment, there are only few comprehensive contributions that provide guidelines for the optimal design of such structures. In this work, a realistic thin film solar cell with almost conformal layers based on a one-dimensional metallic grating back-reflector is investigated through experiment and theory. The external quantum efficiency of the cell is obtained with the aid of both theory and experiment for different angles of incidence and in both polarizations to validate the computational method and to show the impact of guided mode excitation. Different substrate shapes that are compatible with solar cell fabrication are then considered and the effect of geometrical parameters on the short circuit current density of the device is investigated. Calculations show that among the investigated shapes, trinagular gratings with a very sharp slope in one side, so called sawtooth gratings, are the most promising one-dimensional grating for light trapping. Furthermore, the role of material property is discussed specifically in the back-reflector by simulating aluminum and silver backreflectors. It is shown that the blue response of the solar cells is similar almost regardless of the back-reflector material but their red response is viable to change due to variation in resonant properties of the structure.
\end{abstract}

Keywords: solar cell, diffraction grating

\section{INTRODUCTION}

Solar cells can potentially be exploited to provide cost-effective green energy. Thin film silicon is attractive in particular because of the abundance of silicon. However, both in amorphous and microcrystalline cells, the poor electric transport properties necessitate cell thicknesses below the optical absorption length in the red part of the spectrum. Coupling light into the thin film silicon solar cell and trapping light into its absorber layer is, hence, a major objective.

There has been a lot of effort devoted to solving the latter problem using periodic ${ }^{1-5}$ and random ${ }^{6}$ interface textures. Yablonovitch suggested a theoretical upper limit for the effective light path enhancement in the cell based on the two main assumptions: The absorber is weakly absorbing and the scattering is Lambertian at the interfaces ${ }^{7}$. The latter limit is not reached yet in experiment to our knowledge. Actually, it has been proposed that periodic structures can even surpass the mentioned limit in a narrow wavelength range ${ }^{8}$ or in broadband but using thick solar cells ${ }^{4,5}$. However, in thin film solar cells, the absorption enhancement is required to work in a relatively broad spectral range with cell thicknesses of hundreds of nanometers.

In order to evaluate light trapping of a solar cell with textured interfaces, the external quantum efficiency -EQE- is defined as the number of charge collected carriers with respect to the incident photon flux in short-circuit condition. One can approximately calculate EQE using optical simulations as performed in Ref. 9 and Ref. 10. Another well-known figure of merit is the short circuit current density $\left(\mathrm{J}_{\mathrm{sc}}\right)$ which is obtained by integrating the product of EQE and spectral irradiance $\Phi$ of sunlight over the wavelength $\lambda$.

$$
J_{s c}=\int_{\text {solar spectrum }} E Q E(\lambda) \Phi(\lambda) d \lambda
$$

*ali.naqavi@epfl.ch; phone +41 327183 338; http://pvlab.epfl.ch/

SPIE Eco-Photonics 2011: Sustainable Design, Manufacturing, and Engineering Workforce Education for a Green Future, edited by Pierre Ambs, Dan Curticapean, Claus Emmelmann, Wolfgang Knapp, Zbigniew T. Kuznicki, Patrick P. Meyrueis, Proc. of SPIE Vol. 8065, 80650A - (C) 2011 SPIE · CCC code: 0277-786X/11/\$18 · doi: 10.1117/12.883186 
In this work, we present measured photocurrent enhancement of a thin film solar cell with textured interfaces based on a one dimensional (1D) metallic grating, and we compare the results to Rigorous Coupled Wave Analysis (RCWA) predictions using the correct geometry of the interfaces. Then, we apply the modeling to investigate optical properties of the solar cell based on different shapes of back-reflector grating: sinusoidal, sawtooth, perfectly-sawtooth and binary and different back-reflector materials: silver and aluminum. The simulations are performed under normal incidence of light unless specified. Note that in this paper the grating notation is used; i.e. the P polarization refers to the case where the electric field vector is parallel to the grating grooves and $\mathrm{S}$ polarization stands for the case where the magnetic field vector is parallel to the grooves.

\section{EXPERIMENTAL}

The investigated device is a single junction amorphous silicon solar cell grown on a $1 \mathrm{D}$ grating substrate that serves as a textured back reflector. Figure 1 shows a SEM micrograph of the cell. The substrate grating period is $560 \mathrm{~nm}$, its peak to valley is $140 \mathrm{~nm}$ and it is covered with $120 \mathrm{~nm}$ of $\mathrm{Ag}, 60 \mathrm{~nm}$ of $\mathrm{ZnO}$, an n-i-p junction made from amorphous silicon with nominal thicknesses of $13 \mathrm{~nm}, 200 \mathrm{~nm}$ and $17 \mathrm{~nm}$ for the n-Si, i-Si and p-Si respectively. The front contact is made from a $60 \mathrm{~nm}$ sputtered indium-tin oxide (ITO) layer to yield an anti-reflection effect at the wavelength of about $550 \mathrm{~nm}$. Experimental details can be found in Ref. 1.
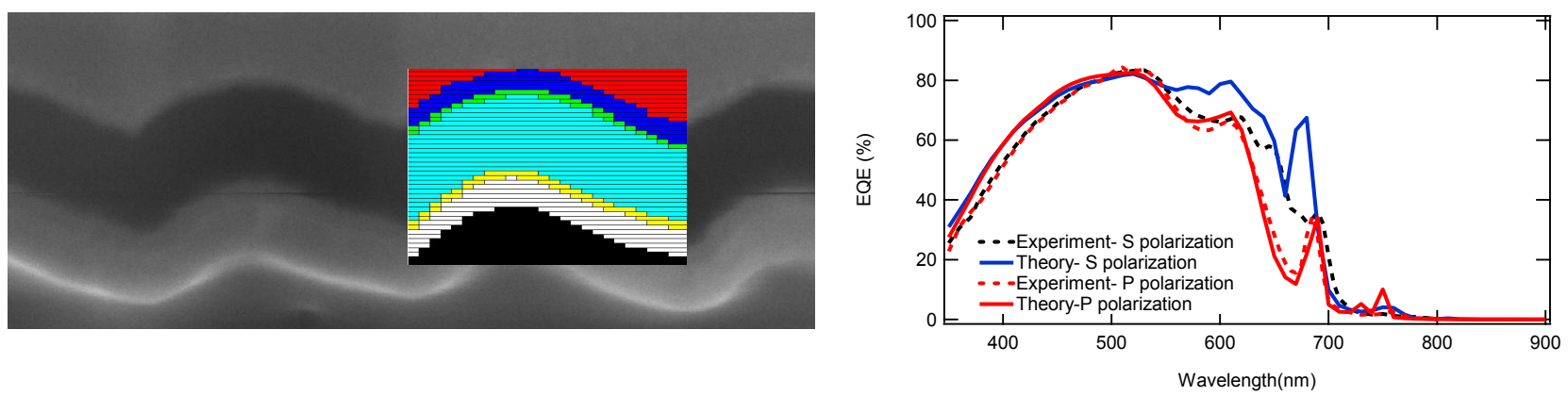

Figure 1. Left: SEM picture of the experimental sample. The plot shows the approximate structure used in simulations of S polarization. Right: Comparison of EQE obtained from experiment and theory in both polarizations under normal incidence

\section{OBSERVATION OF GUIDED MODES AND THEIR ANGULAR VARIATION}

Most device simulations consider ideal structures like perfect sinusoidal or binary gratings and the layers are considered perfectly conformal. However, there are always deviations from these assumptions practically. We have considered such deviations by fitting our model to the SEM image as depicted in Figure 1. Details about simulation can be found in Ref. 9. The individual layer thicknesses are adapted to $60,15,180,20$, and $70 \mathrm{~nm}$ for ITO, p-Si, i-Si, n-Si and the ZnO buffer layer, respectively to reach agreement between experiment and theory and silver is assumed to be semi-infinite.

The experimental and theoretical values of EQE vs. wavelength are compared in Figure 1 with the assumption of normal illumination. Similar EQE curves can be obtained if the incident angle is changed. In the latter case, guided modes will shift in wavelength since the phase matching condition will not be satisfied at the same wavelength. Figure 2 illustrates EQE curves obtained for different angles of incidence from $-50^{\circ}$ to $+50^{\circ}$ by steps of $10^{\circ}$, showing that the main features of the experiment are reproduced by the theoretical results. The EQE peaks and their shift as a function of incident angle can be explained by guided mode excitation. Better agreement of theory and experiment in P polarization can be explained by finer slicing and innate faster convergence in $\mathrm{P}$ polarization. To evaluate the amount of enhancement, we compare the sample with a cell with flat interfaces based on their $\mathrm{J}_{\mathrm{sc}}$ values. Experimental values of $\mathrm{J}_{\mathrm{sc}}$ are $11.8 \mathrm{~mA} / \mathrm{cm}^{2}, 12.95 \mathrm{~mA} / \mathrm{cm}^{2}$ and $10.63 \mathrm{~mA} / \mathrm{cm}^{2}$ for the $\mathrm{P}$ - and $\mathrm{S}$ polarizations and for the flat substrate respectively. The corresponding theoretical values are $11.65 \mathrm{~mA} / \mathrm{cm}^{2}, 13.60 \mathrm{~mA} / \mathrm{cm}^{2}$ and $10.97 \mathrm{~mA} / \mathrm{cm}^{2}$ respectively.

Geometrical parameters such as layer thicknesses and the depth and period of grating can dramatically affect the $\mathrm{EQE}$. Up to the wavelengths of about $550 \mathrm{~nm}, \mathrm{EQE}$ is dominated by absorption loss in the window layers, i.e. p-Si and ITO. At longer wavelengths, the response benefits from a Fabry-Perot interference fringe at about $600 \mathrm{~nm}$ and several guided modes the most prominent of which is observed at $690 \mathrm{~nm}$ for perpendicular incidence and $\mathrm{P}$ polarization. Both interference fringe and the guided modes are significantly influenced by the thickness of the silicon layers. Because of the high refractive index, thicker layers generally move all features towards longer wavelengths. Changing the grating 
depth normally does not have a major impact on the positions of guided mode resonances but can strengthen the resonance in deeper gratings. Increasing the grating period leads to red-shift of the features.

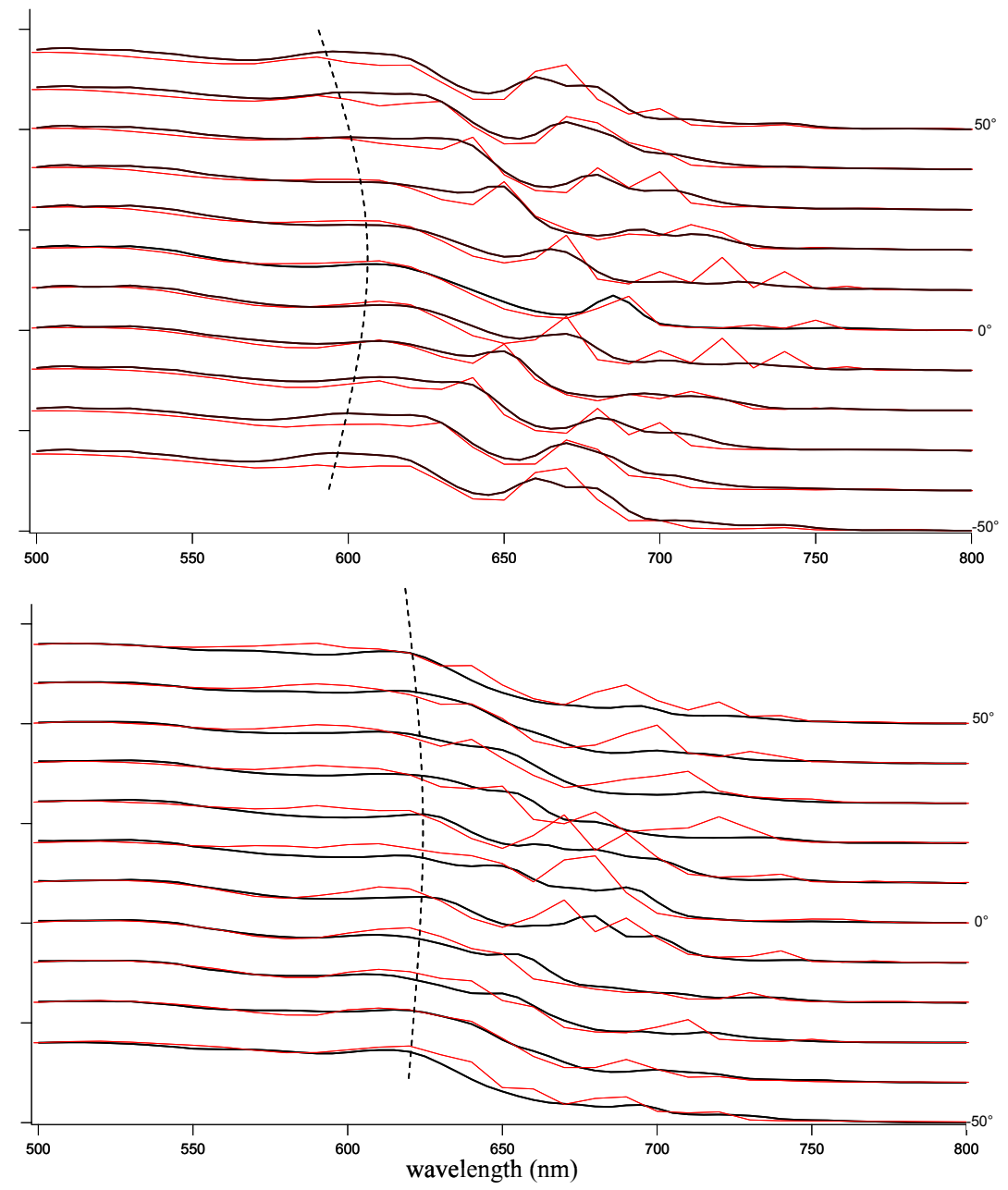

Figure 2. Angular variations of EQE vs wavelength. The red curves are theoretical results and the black curves are the experimental ones. (up): P polarization, (bottom): S polarization. The dashed line shows the small angular variation of interference.

\section{RESULTS AND DISCUSSION}

\subsection{Impact of backreflector shape}

In this section, four different general back-reflector shapes based on 1D gratings are investigated and their impact on the absorption enhancement is discussed. In Figure 3, the mentioned generic shapes are plotted and the free parameters are demonstrated by arrows.

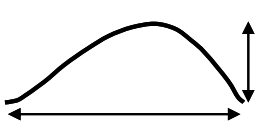

(a)

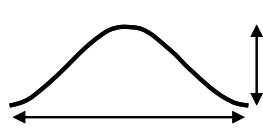

(b)

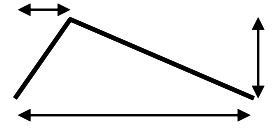

(c)

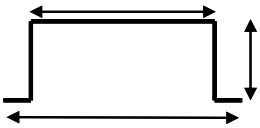

(d)

Figure 3. Four different types of back-reflector grating; a: sawtooth (experimental sample), b: perfect sinusoidal, c: perfect sawtooth and d: binary. Only one period of the back-reflector is shown and the parameters that change are exhibited in each case by arrows. 
Two geometries are considered first: the sawtooth grating with realistic interfaces -Figure 1 and Figure 3(a)-, but scaled in depth and period, and a perfect sinusoidal back-reflector with conformal layers -Figure 3 (b). The thicknesses of layers on the sinusoidal grating are assumed to be the same as the respective thicknesses mentioned in the previous section for the experimental sample. The $\mathrm{J}_{\mathrm{sc}}$ obtained through simulation is plotted in Figure 4 for both polarizations and for both shapes. Also, the unpolarized $\mathrm{EQE}$-which can be obtained through averaging the results of the two polarizations- is depicted in Figure 5 for the sake of comparison. Overall, the sawtooth grating exhibits higher photocurrents in a wider parameter space than the sinusoidal grating, and there is a better overlap of high- $\mathrm{J}_{\mathrm{sc}}$ regions for the two polarizations. In our simulations, maximal $J_{\mathrm{sc}}$ values of $13.2 \mathrm{~mA} / \mathrm{cm}^{2}$ and $13.0 \mathrm{~mA} / \mathrm{cm}^{2}$ are obtained for the sawtooth and the sinusoidal grating.
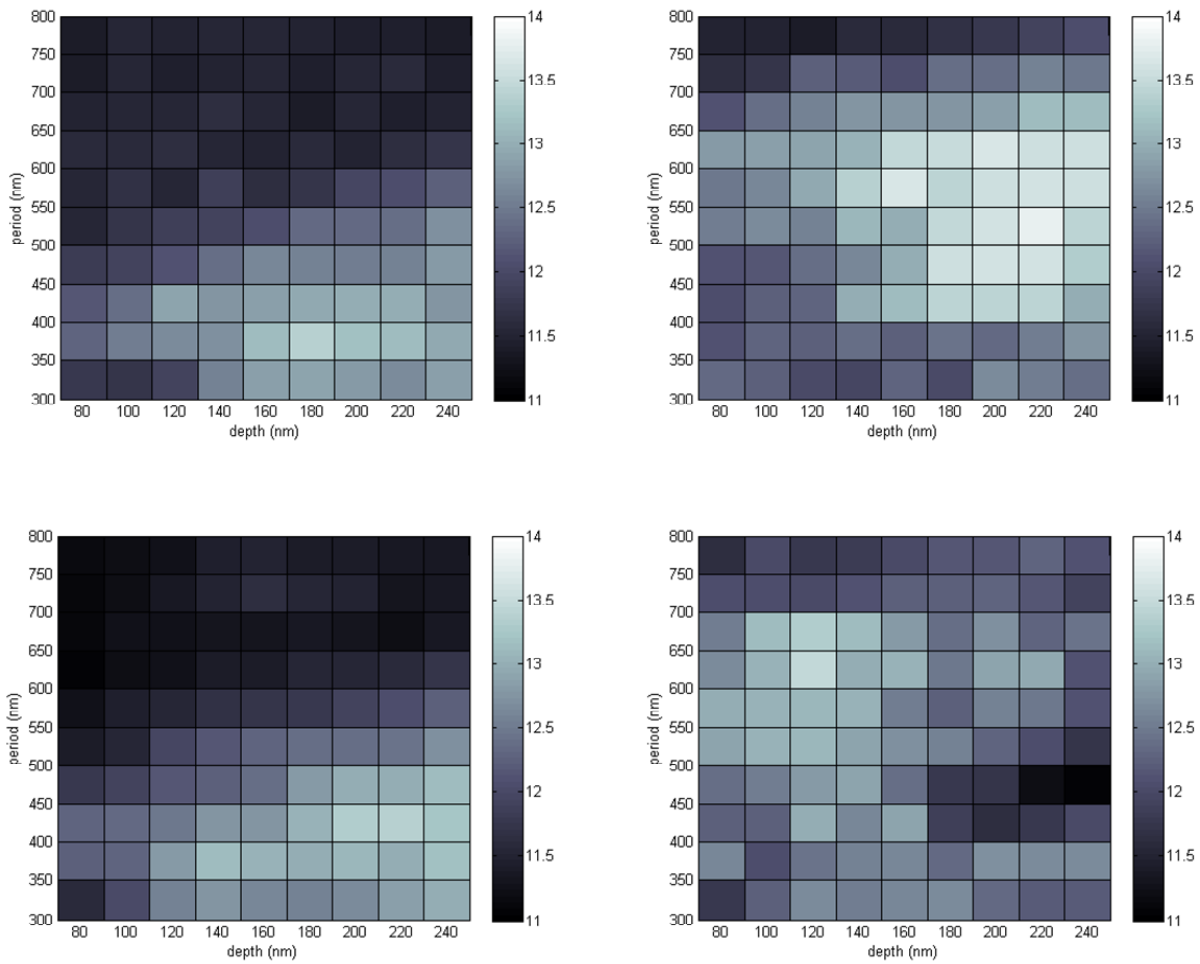

Figure 4. Short circuit current density $\left(\mathrm{mA} / \mathrm{cm}^{2}\right)$ variation vs period $(\mathrm{nm})$ and depth $(\mathrm{nm})$ of grating for P polarization (left) /S polarization (right). The metallic back-reflector is a sawtooth grating as in the experiment (up)/ sinusoidal grating (bottom).
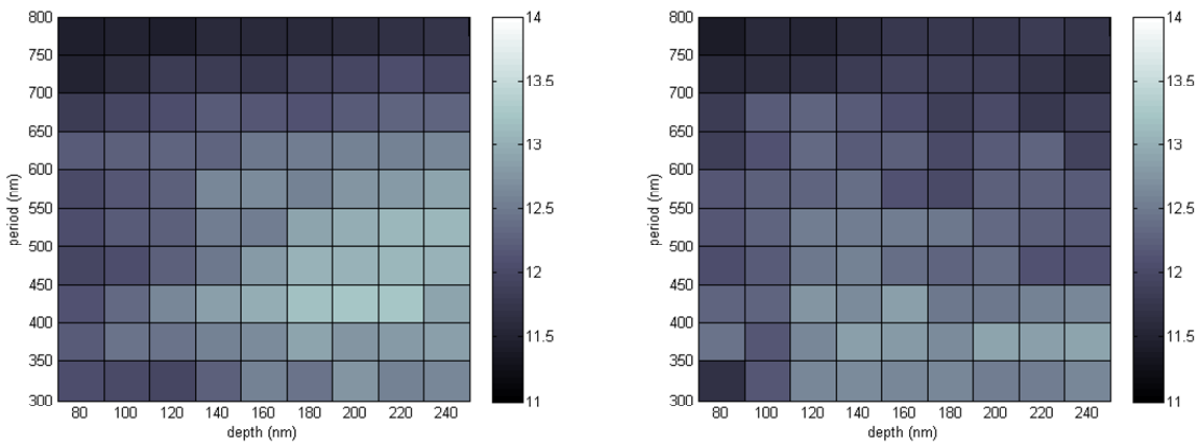

Figure 5. Short circuit current density $\left(\mathrm{mA} / \mathrm{cm}^{2}\right)$ variation vs period $(\mathrm{nm})$ and depth $(\mathrm{nm})$ of grating under unpolarized light for experimental sample (left) and sinusoidal grating (right). 
We also consider an ideally sawtooth grating shape - Figure 3 (c). Based on our simulations the pure sawtooth grating can result in higher $\mathrm{J}_{\mathrm{sc}}$ if one of its sides is as steep as possible. We simulated such a structure with the sawtooth edge positioned at $10 \%$ of the period, assuming the same thickness of layers as in the previous part. The results are shown in Figure 6. In the $\mathrm{P}$ polarization, $\mathrm{J}_{\mathrm{sc}}$ values are not as high as the previous cases but the $\mathrm{S}$ polarization $\mathrm{J}_{\mathrm{sc}}$ takes larger values. Furthermore, the strong overlap of the two polarizations can be used beneficially to obtain higher $\mathrm{J}_{\mathrm{sc}}$ under unpolarized light. Our conclusion is that for the perfectly sawtooth grating, the short circuit current density depends more on the depth of the grating especially in S polarization. The experimental sample represents a compromise between high current densities and appropriate polarization overlap.

We also compare the results obtained for an ideal sawtooth -Figure 3 (c)- grating back-reflector with the case of a binary one - Figure 3 (d). As before, the layers are considered conformal with the previously mentioned thicknesses. In the case of sawtooth grating, the high- $\mathrm{J}_{\text {sc }}$ regions in the two polarizations exhibit a strong overlap but the binary shape does not provide this feature. In the latter case, there is no global trend in P polarization to maximize the $\mathrm{J}_{\mathrm{sc}}$ for specific grating parameters, i.e. there is more than one region of high $\mathrm{J}_{\mathrm{sc}}$ as previously observed ${ }^{11}$. Such a splitting of high- $\mathrm{J}_{\mathrm{sc}}$ regions is not observed in the case of $\mathrm{S}$ polarization. Figure 7 shows the impact of different parameters on the unpolarized $\mathrm{J}_{\mathrm{sc}}$ of the two latter gratings. Note that Figure 7 shows only a cut of the complete results in the optimal duty cycle/ sawtooth edge position. It is observed that the maximal $J_{s c}$ values are almost the same for both sawtooth and binary gratings but $\mathrm{J}_{\mathrm{sc}}$ is more sensitive to geometrical parameters in the case of binary grating.

Altogether, cells with sinusoidal back-reflectors will suffer from poor overlap of optimal regions in P- and S polarizations. A global optimum is lacking for the binary grating besides the poor overlap of optimal regions in the two polarizations. A sawtooth back-reflector, if designed correctly, benefits from both a global maximum and very good overlap of the two polarizations. S polarization produces higher $\mathrm{J}_{\mathrm{sc}}$ values for all of the cases studied here. Again we emphasize that the conclusions in this section are obtained for normal incidence.
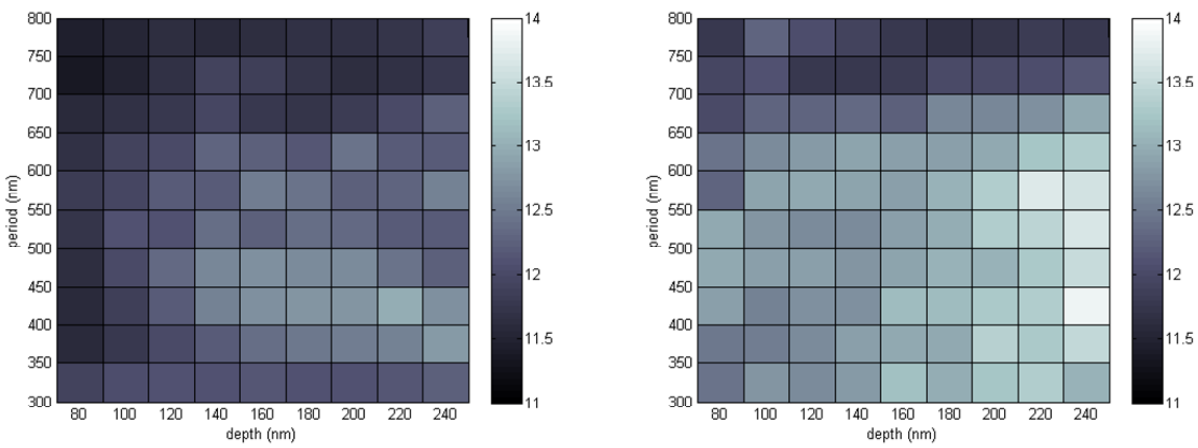

Figure 6. Variation of $\mathrm{J}_{\mathrm{sc}}\left(\mathrm{mA} / \mathrm{cm}^{2}\right)$ vs period $(\mathrm{nm})$ and depth $(\mathrm{nm})$ of grating for $\mathrm{P}$ polarization (left) /S polarization (right). The metallic back-reflector is a perfect sawtooth grating with sawtooth edge at 0.1 of the period.
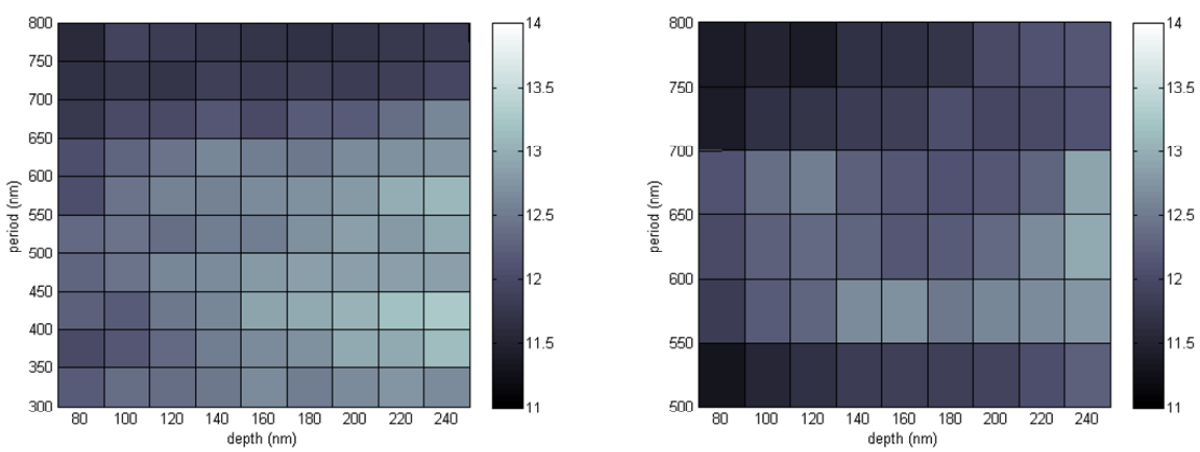

Figure 7. Variation of $\mathrm{J}_{\mathrm{sc}}\left(\mathrm{mA} / \mathrm{cm}^{2}\right)$ vs period and depth of grating under unpolarized light for sawtooth grating with sawtooth edge positioned at 0.1 of the period (left) and for binary grating with duty cycle of 0.9 (right). Note that periods below $500 \mathrm{~nm}$ are not feasible for the binary grating because the sidewalls would touch for the chosen layer thicknesses. 


\subsection{Behaviour in the blue part of the spectrum}

Optical losses in the solar cells can be categorized based on their origin: reflection and parasitic absorption. Reflection from the solar cell front interface can be reduced using an anti-reflection (AR) coating layer. In our experimental sample, the ITO layer performs the AR task and it is basically designed for the wavelength of $550 \mathrm{~nm}$. Using interface textures is another way to reduce reflection as previously reported by Heine and Morf ${ }^{3}$ and Haase and Stiebig ${ }^{10}$.

Figure 8 shows the ratio of the power reflected to the incident power for some different grating depths. It is revealed from Figure 8 that reflection can cause at most a loss of about $15 \%$ in the plotted wavelength range but EQE drop is much more for short wavelengths -Figure 1. Hence, reflection cannot be the major loss mechanism in short wavelengths. Actually, if we take into account perfect AR effect, i.e. if we assume that all of the incident power is coupled into the structure, there is still a huge portion of energy which is absorbed in the window layers. Figure 9 shows the EQE plots for two different representative grating depths of 30 and $150 \mathrm{~nm}$. It also includes two other approximations of EQE which consider only absorption in window layers. In the latter approximations, reflection effects are ignored and it is assumed that the layers are flat to ease computation. Figure 9 reveals that parasitic absorption in the p-Si layer is responsible for the major part of loss at short wavelengths.

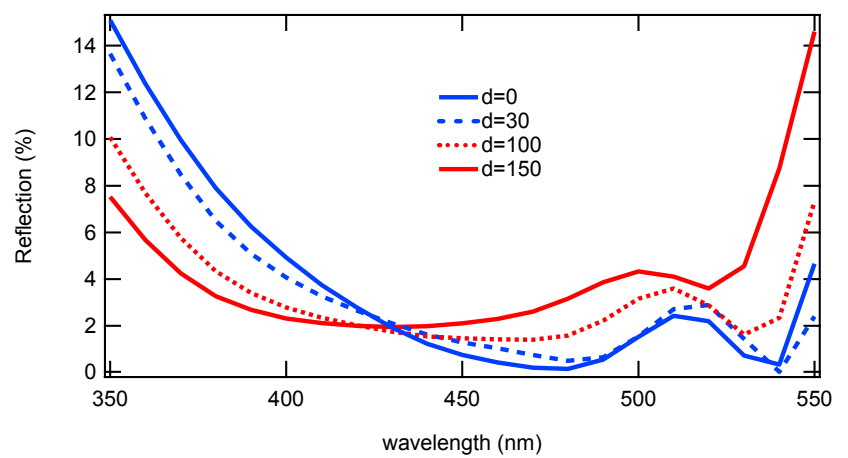

Figure 8. Reflected power normalized to the incident power (\%) for different grating depths. The geometry is similar to the experimental sample but scaled in depth.
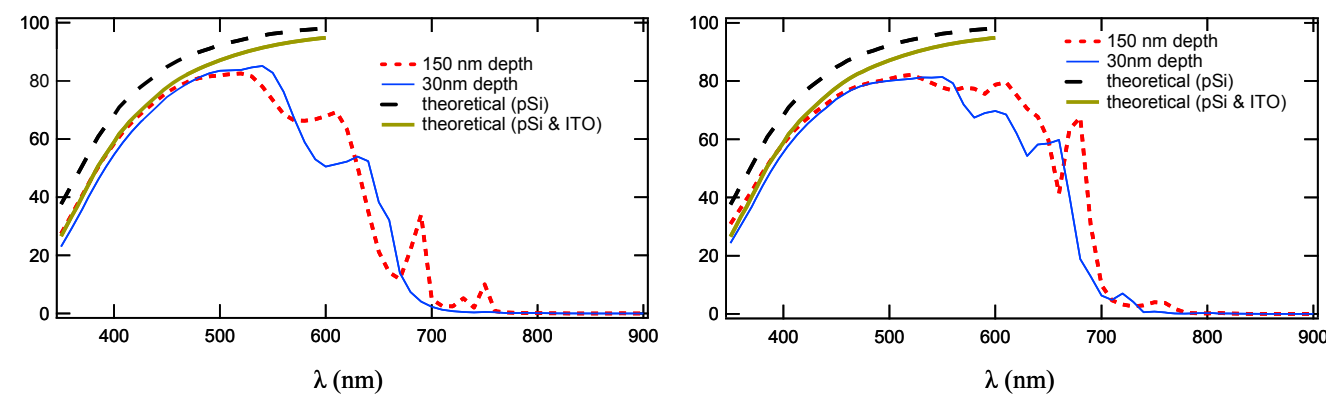

Figure 9. EQE for two different grating back-reflectors mentioned in section 4.2 for both $\mathrm{P}$ (left) and $\mathrm{S}$ polarization (right). The EQE approximations considering the absorption in the $\mathrm{p}-\mathrm{Si}$ alone, and in $\mathrm{p}-\mathrm{Si}$ and ITO layer are illustrated between 350 and $600 \mathrm{~nm}$ by dashed and solid lines respectively.

\subsection{Impact of back-reflector material}

Up to now, all the presented examples were performed with a silver back-reflector. From an economical point of view, it would be desirable to replace the silver with a less expensive material e.g. aluminum. Hence, in this section, we repeat the simulations for the case when silver in the back-reflector is replaced with aluminum. For the sake of being in more agreement with experiment, we use the geometry of the experimental sample.

Figure 10 shows the calculated EQE for the two cases: the experimental sample and its counterpart with aluminum back-reflector. As observed, the EQE of both cases is similar in the blue part of the spectrum as predicted by the discussion in section 4.2. At longer wavelengths, the performance of the aluminum back-reflector is at least as good as 
the silver one. According to our simulations, similar conclusions can be made for different thicknesses of $\mathrm{ZnO}$ buffer layer. Associated results are not shown here for the sake of brevity. As shown in Figure 10, removing the $\mathrm{ZnO}$ layer will lead to a harsh plasmon resonance dip in EQE in the case of silver while aluminum does not exhibit a plasmon resonance in the investigated range of wavelengths. From purely optical considerations, the $\mathrm{ZnO}$ buffer layer could therefore be removed from the cells with aluminum back-reflector. Experimentally, solar cells with aluminum back-reflectors show worse performance ${ }^{12}$ and the reasons behind this have to be investigated.
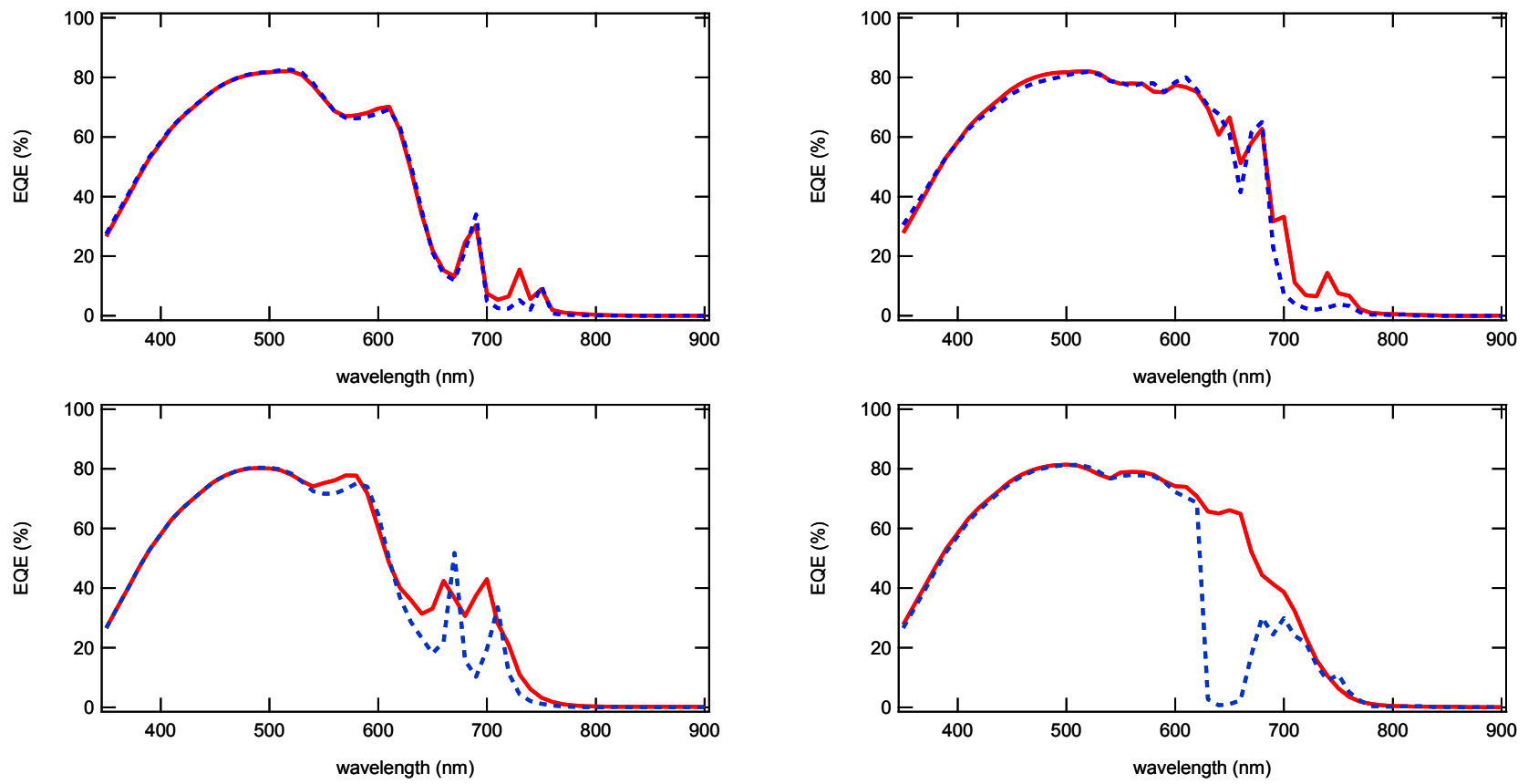

Figure 10. Comparison of EQE for the silver (dashed) and aluminum (solid) back-reflectors in P- (left) and S polarization (right). The geometry is assumed to be the same as experimental sample (top) and the same geometry when the $\mathrm{ZnO}$ layer is removed.

\section{CONCLUSIONS}

A real thin film silicon solar cell grown on a one-dimensional metallic grating is investigated experimentally and theoretically. Guided modes are used to improve the photocurrent and their angular variation is observed in both theory and experiment. The impact of geometrical parameters such as the period and the height of the back-reflector grating is discussed for different grating patterns. According to the simulations, sawtooth gratings can provide high photocurrent in a wide parameter range for normal incidence but the other considered shapes suffer from either lack of high photocurrent in both polarizations simultaneously or high sensitivity to geometrical parameters. The role of material property is then discussed specifically in the front layers and in the back-reflector. It is found that parasitic absorption in the front layers is the main loss mechanism at short wavelengths. Also, according to our simulations, aluminum seems a wise choice of material to replace silver in the back-reflector since it can provide higher values of photocurrent than silver. Unfortunately, in practical work, silver back-reflectors lead to higher short circuit current when compared with aluminum.

\section{ACKNOWLEDGEMENT}

We thankfully acknowledge Dr. Aïcha Hessler and Caroline Calderone from the CIME at EPFL for cross-section microscopy and Dr. Rudolf Morf and David Gablinger from the PSI Villigen for stimulating discussions. This work is funded by the Swiss National Science Foundation under project number 200021_125177/1. 


\section{REFERENCES}

[1] Söderström, K., Haug, F.-J., Escarré, J. et al., "Photocurrent increase in nip thin film silicon solar cells by guided mode excitation via grating coupler," Appl. Phys. Lett. 96, 213508 (2010).

[2] Isabella, O., Moll, F., Krč, J. et al., "Modulated surface textures using zinc oxide films for solar cells applications," Phys. Status Solidi A 207(3), 642-646 (2010).

[3] Heine, C. and Morf, R., "Submicrometer gratings for solar energy applications," Appl. Opt. 34(14), 2476-2482 (1995).

[4] Yu, Z., Raman, A. and Fan, S., "Fundamental limit of nanophotonic light-trapping in solar cells," Optics for Solar Energy, OSA Technical Digest (CD) PDSWB1 (2010).

[5] Yu, Z., Raman, A. and Fan, S., "Fundamental limit of light trapping in grating structures," Opt. Express 18(S3), A366-A380 (2010).

[6] Söderström, T., Haug, F.-J., Niquille, X. et al., "TCOs for nip thin film silicon solar cells," Prog. Photovoltaics Res. Appl. 17(3), 165-176 (2009).

[7] Yablonovitch, E. and Cody, G. D., "Intensity enhancement in textured optical sheets for solar cells," IEEE Trans. Electron Devices 29(2), 300-305 (1982).

[8] Sheng, P., Bloch, A. and Stepleman, R., "Wavelength selective absorption enhancement in thin film solar cells," Appl. Phys. Lett. 43, 579-581 (1983).

[9] Naqavi, A., Söderström, K., Haug, F.-J. et al., "Understanding of photocurrent enhancement in real thin film solar cells: towards optimal one-dimensional gratings," Opt. Express, 19(1), 128-140 (2011).

[10]Haase, C. and Stiebig, H., "Thin-film silicon solar cells with efficient periodic light trapping texture," Appl. Phys. Lett. 91, 061116 (2007).

[11]Zanotto, S., Liscidini, M. and Andreani, L., "Efficiency enhancement in thin-film silicon solar cells with a photonic pattern," Optical Nanostructures for Photovoltaics, OSA Technical Digest (CD) PTuB1 (2010).

[12] Kothandaraman, C., Tonon, T., Huang, C. et al., "Improvement of a-Si:H PIN devices using zinc oxide based back-reflectors," Mat. Res. Soc. Symp 219, 475-480 (1991). 